\title{
Comparative Analysis of Conventional, P, PI, PID and Fuzzy Logic Controllers for the Efficient Control of Concentration in CSTR
}

\author{
Farhad Aslam \\ Department of Electrical \& Instrumentation Engineering \\ Thapar University, Patiala, Punjab, India
}

\author{
Gagandeep Kaur \\ Department of Electrical and Instrumentation Engg. \\ Thapar University, Patiala, Punjab, India
}

\begin{abstract}
All the industrial process applications require solutions of a specific chemical strength of the chemicals or fluids considered for analysis. Such specific concentrations are achieved by mixing a full strength solution with water in the desired proportions. In this paper the control the concentration of one chemical with the help of other has been analyzed. This paper features the influence of different controllers like P, PI, PID and Fuzzy logic controller upon the process model. Model design and simulation are done in MATLAB SIMULINK, using fuzzy logic toolbox. The concentration control is found better controlled with the addition of fuzzy logic controller instead of PID controller solely. The improvement of the process has been observed.
\end{abstract}

Keywords: Fuzzy Logic (FL), PID Control, Chemical Concentration, Mamdani Fuzzy model, CSTR

\section{INTRODUCTION}

Chemical reactors often have significant heat effects, so it is important to be able to add or remove heat from them. In a CSTR (continuously stirred tank reactor) the heat is add or removed by virtue of the temperature difference between a jacked fluid and the reactor fluid. Often, the heat transfer fluid is pumped through agitation nozzle that circulates the fluid through the jacket at a high velocity. The reactant conversion in a chemical reactor is a function of a residence time or its inverse, the space velocity. For a CSTR, the product concentration can be controlled by manipulating the feed flow rate, which change the residence time for a constant chemical reactor.

A proportional controller could lead to offset between the desired set point and the actual output. This is because the process input which is controller output and the process output come to new equilibrium values before error goes down to zero. Now to make the controller output proportional to the integral of the error desired compensation is to be provided. This is known as the proportional integral control. As long as there continuous to be an error signal to the controller, the controller output will continue to change. Therefore, the integral of error forces the error signal to zero. Now add one more term that accounts for current rate of change i.e. derivative of the error. This is known as proportional integral derivative control. Using knowledge of the error helps the controller to predict where in future the error is heading and compensate for it.

Fuzzy systems are universal approximates. Fuzzy controlled systems models do not require any certain model for implementation of system under consideration. These proofs stem isomorphism between an abstract algebra and linear algebra and the structure of a Fuzzy system, which comprised of an implication between actions and conclusion as antecedents and consequents. Abstract algebra incorporates systems or models dealing with groups, fields and rings. Linear algebra incorporates system models dealing with vector spaces, state vector and transition matrices. The primary benefit of fuzzy system theory is to approximate system behavior where numerical functions or analytical functions do not exist. Hence, Fuzzy systems have high potential to understand the very systems that are devoid of analytical formulations in a complex System. Complex systems can be new systems that have not been tested, they can involve with the human conditions such as biological or medical systems. The ultimate goal of the fuzzy logic is to form the theoretical foundation for reasoning about the imprecise reasoning, such reasoning is known as approximate reasoning.

In this paper, CSTR has been used to mix ethylene oxide with water to make ethylene glycol. Here the purpose is to control the concentration of ethylene glycol with the help of concentration of ethylene oxide. But undershoot and overshoot come in the considered system while performing in a conventional way. But after implementation of a PID controller to the process, removing of those shoots can be seen but still the output is unstable. So finally fuzzy logic controller is used to achieve a desirable output.

\section{CASE STUDY}

In this paper, CSTR has been considered in which concentration of two chemicals is controlled for better results, the chemical ' $\mathrm{X}$ ' and ' $\mathrm{Y}$ ' and the byproduct is ' $\mathrm{Z}$ '. Ethylene oxide $(\mathrm{X})$ is reacted with water $(\mathrm{Y})$ in a continuously stirred tank reactor (CSTR) to form ethylene glycol (Z).Assume that the CSTR is manipulated at a constant temperature and that the water is in large excess. The stoichiometric equation is

$\mathrm{X}+\mathrm{Y}=\mathrm{Z}$

The reactant conversion in a chemical reactor is a function of residence time or its inverse, the space velocity. For an isothermal CSTR, the product concentration can be controlled by manipulated the feed flow rate, which change the residence time (for a constant volume reactor). It is convenient to work in molar units when writing components balances, particularly if chemical reaction is involved. Let $\mathrm{CX}$ and $\mathrm{CZ}$ represent the molar concentration of $\mathrm{X}$ and $\mathrm{Z}$ (mol/volume).

$\underline{\mathrm{dVC}} \underline{\mathrm{X}}=\mathrm{F}_{\mathrm{i}} \mathrm{C}_{\mathrm{Xi}}-\mathrm{FC}_{\mathrm{X}}+\mathrm{Vr}_{\mathrm{X}} \ldots \ldots \ldots \ldots . .$.
$\mathrm{dt}$
$\frac{\mathrm{dVCZ}}{\mathrm{dt}}=-\mathrm{FC}_{\mathrm{z}}+\mathrm{Vr}_{\mathrm{Z}} \ldots \ldots \ldots \ldots \ldots \ldots$


Where $r_{X}$ and $r_{Z}$ represent rate of generation of species $X$ and $Z$ per unit volume, and $\mathrm{C}_{\mathrm{Xi}}$ represents the inlet concentration of species X. If the concentration of the water change than the reaction rate is second order with respect to the concentration of Ethylene oxide

$r_{X}=-k_{1} C_{X}-k_{3} C_{X}^{2}$

Where $\mathrm{k}_{1}, \mathrm{k}_{2} \& \mathrm{k}_{3}$ are the reaction rate constants and the minus sign indicate that $\mathrm{X}$ is consumed in the reaction. Each mole $\mathrm{X}$ reacts with a mole of $\mathrm{Y}$ and produces one mole of $\mathrm{Z}$, so the rate of generation of $\mathrm{Z}$ is

$\mathrm{r}_{\mathrm{Z}}=\mathrm{k}_{1} \mathrm{C}_{\mathrm{X}}-\mathrm{k}_{2} \mathrm{C}_{\mathrm{Z}}$

Expanding the left hand side of equation (1)

$\frac{\mathrm{dVC}_{\mathrm{X}}}{\mathrm{dt}}=\frac{\mathrm{V} \underline{\mathrm{dC}}_{\mathrm{X}}}{\mathrm{dt}}+\underset{\mathrm{dt}}{\mathrm{C}_{\mathrm{X}} \frac{\mathrm{dV}}{}}$

Combining eq (1) \& (5)

$\frac{\mathrm{dC}}{\mathrm{dt}} \underline{\underline{\mathrm{X}}}=\frac{\mathrm{F}_{\mathrm{i}}}{\mathrm{V}}\left(\mathrm{C}_{\mathrm{Xi}}-\mathrm{C}_{\mathrm{X}}\right)-\mathrm{k}_{1} \mathrm{C}_{\mathrm{X}}-\mathrm{k}_{3} \mathrm{C}_{\mathrm{X}}^{2}$

Similarly,

$\frac{\mathrm{dC}}{\mathrm{dt}} \underline{Z}=-\frac{\mathrm{F}}{\mathrm{V}} \mathrm{C}_{\mathrm{Z}}+\mathrm{k}_{1} \mathrm{C}_{\mathrm{X}}-\mathrm{k}_{2} \mathrm{C}_{\mathrm{Z}}$

\section{PROBLEM FORMULATION}

The linear space model or case study of CSTR is given by

$\mathrm{x}=\mathrm{Ax}+\mathrm{Bu}$

$\mathrm{y}=\mathrm{Cx}+\mathrm{Du}$

Where the states, inputs and output are in deviation variable form.

The first input (dilution rate) is manipulated and the second (feed concentration of $\mathrm{A}$ ) is a disturbance input. Linearization of the two modeling equations (from equation (6) \& (7)) at steady state solution to find the following state space matrices is done:

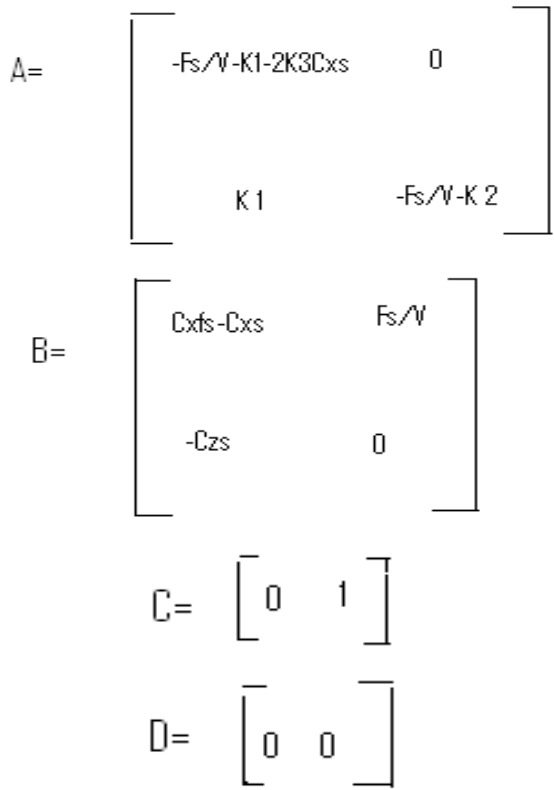

For the particular reaction under consideration, the rate constants are $\mathrm{k}_{1}=5 / 6 / \mathrm{min} \quad \mathrm{k}_{2}=5 / 3 / \mathrm{min} \quad \mathrm{k}_{3}=1 / 6$ $\mathrm{mol} /$ litre.min Based on the steady state operating point of $\mathrm{C}_{\mathrm{Xs}}=3 \mathrm{gmol} / \mathrm{liter}, \mathrm{C}_{\mathrm{Zs}}=1.117 \mathrm{gmol} /$ liter and $\mathrm{F}_{\mathrm{s}} / \mathrm{V}=0.5714$ $\mathrm{min}^{-1}$. The state model is

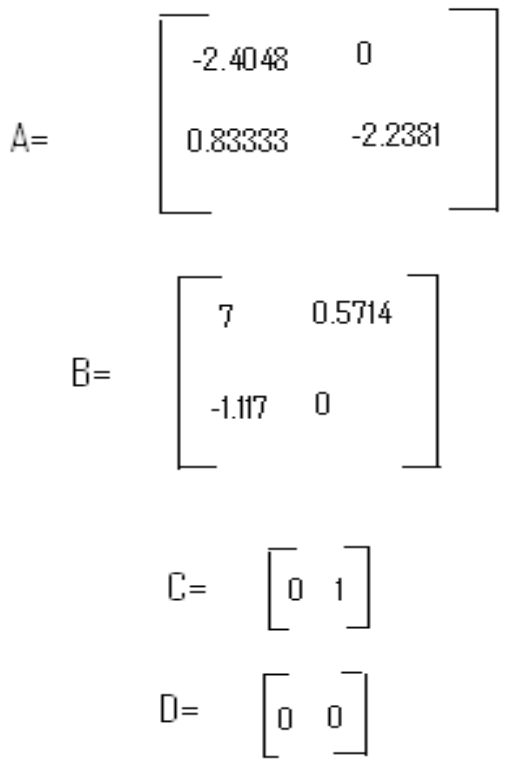

The manipulated input output process transfer function $\mathrm{G}(\mathrm{s})$ $=\mathrm{C}(\mathrm{s} \boldsymbol{I}-\mathbf{A})^{-1} \mathrm{~B}$ is calculated with the help of Matlab.

$\mathrm{G}_{\mathrm{p}}(\mathrm{s})=\frac{-1.117 \mathrm{~s}+3.1472}{\mathrm{~s}^{2}+4.6429 \mathrm{~s}+5.3821}$

It is desired to produce 100 million pounds per day of ethylene glycol. The feed stream concentration is $1.0 \mathrm{lbmol} / \mathrm{ft}^{3}$ and an $80 \%$ conversion of ethylene oxide has been to be determined reasonable. Since $80 \%$ of ethylene oxide is converted to ethylene glycol, the ethylene glycol concentration is $0.8 \mathrm{lbmol} / \mathrm{ft}^{3}$. In this process it is seen that the process has inverse response with delay time as well as overshoot. To overcome this problem and to obtain the desired response, the use of P, PI and PID controller. For that the controller parameters are calculated. The desired parameters for the PID controller are the proportional gain $\left(\mathrm{K}_{\mathrm{P}}\right)$, integral gain $\left(\mathrm{K}_{\mathrm{I}}\right)$ and the differential gain $\left(\mathrm{K}_{\mathrm{D}}\right)$. Firstly, find and solve for the characteristic equation of the process which is given by

$\mathrm{s}^{2}+\left(4.6429-1.117 \mathrm{k}_{\mathrm{c}}\right) \mathrm{s}+\left(5.3821+3.1472 \mathrm{k}_{\mathrm{c}}\right)=0$

... (12)

Where $\mathrm{k}_{\mathrm{c}}$ is the critical (ultimate) gain. The value of $\mathrm{k}_{\mathrm{c}}$ can be calculated by the Routh Hurwitz criterion and the other parameters can be calculated by the Ziegler Nichols tuning method. The values of these parameters are

$K_{\mathrm{P}}=0.1 \mathrm{~K}_{\mathrm{I}}=0.2, \mathrm{~K}_{\mathrm{D}}=\mathbf{0 . 2}$

By putting these values in the simulink PID controller, the response for the step input is obtained. We see that the output have no overshoot but a little inverted response and also its settling time and rise time is little bit more. That is not the desired response. Now for the better control, the Fuzzy logic controller is used. When we connect a Fuzzy logic controller, then we require a multiplexer to give input to the controller. The inputs to the controller are error (difference of the set point and output) and feedback output (output as the feedback). Now construct the membership function for the inputs and the output taking triangular memberships. 
In this paper, the input is unit step input. In this process, the $80 \%$ of the ethylene oxide converted in to the ethylene glycol (output is $80 \%$ of the input). Thus the range for the output is $[0-0.8]$. The second input is error and its range is $[0-0.2]$. Using these values, make fuzzy rules in the fuzzy rule base editor and observe the response that there is no inverted response, no overshoot, no undershoot, rise time and settling time are reduced to a negligible value from our response.

\section{SIMULATION, TESTING AND RESULTS}

The process is represented by the transfer function given in fig. 1, and fig. 2 depicts the output of the process.

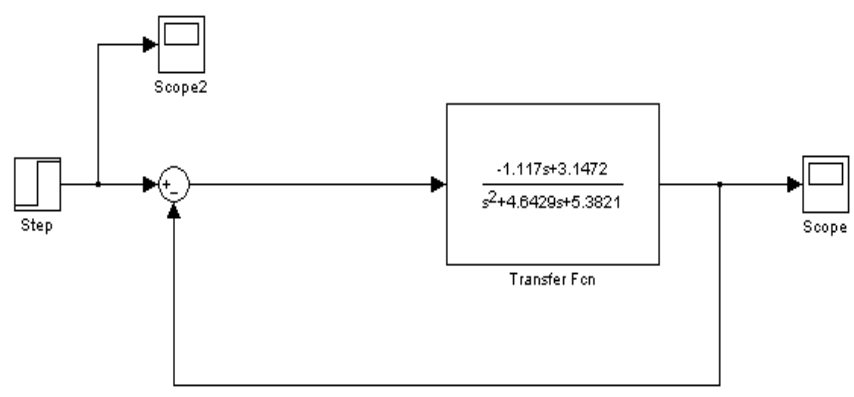

Fig. 1: Process model

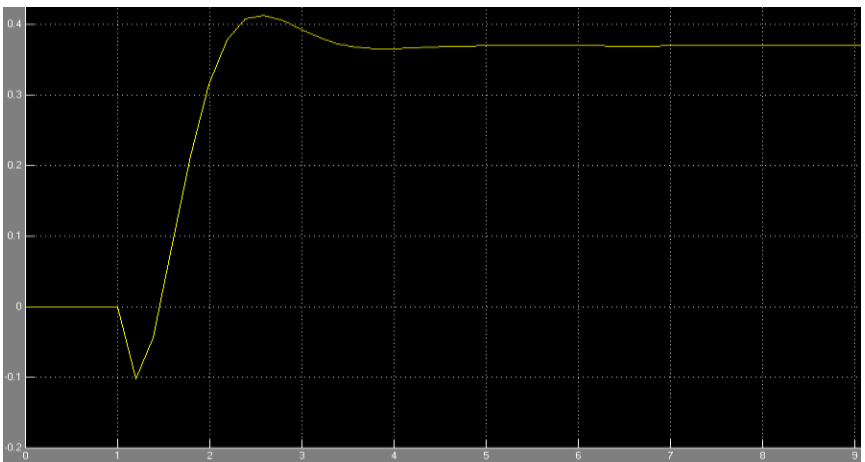

Fig. 2: Time response of uncontrolled process

When there is no control to the process, there is some time delay and inverted response and also the response is settled below the desired magnitude.

The process with $\mathrm{P}$ controller is shown in the fig. 3, and fig.4 depicts the output of the process. Here $K_{P}=100$

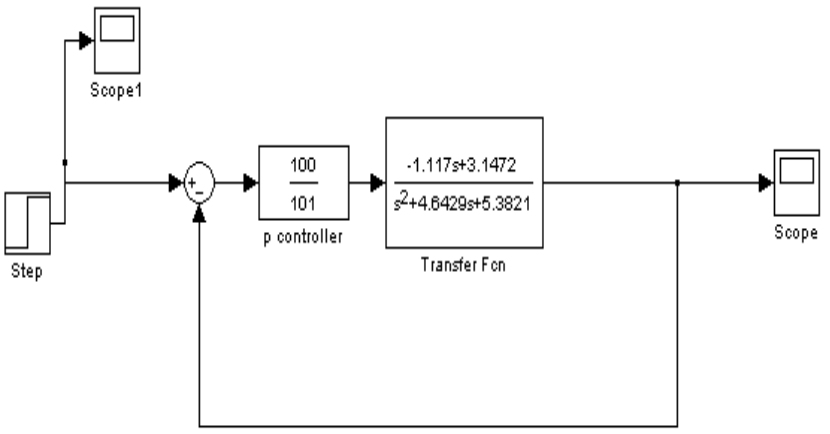

Fig. 3: Process model with P controller

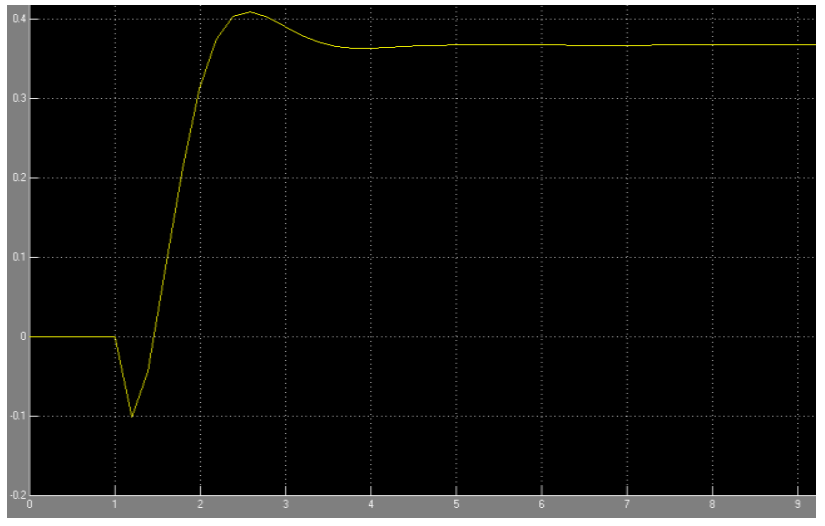

Fig. 4: Time response with $P$ controller

By using $\mathrm{P}$ controller there is not much effect to the output response as compared to uncontrolled process.

The process with PI controller is shown in the fig. 5, and fig. 6 depicts the output of the process.

Here $K_{P}=100$ and $K_{I=} 100$

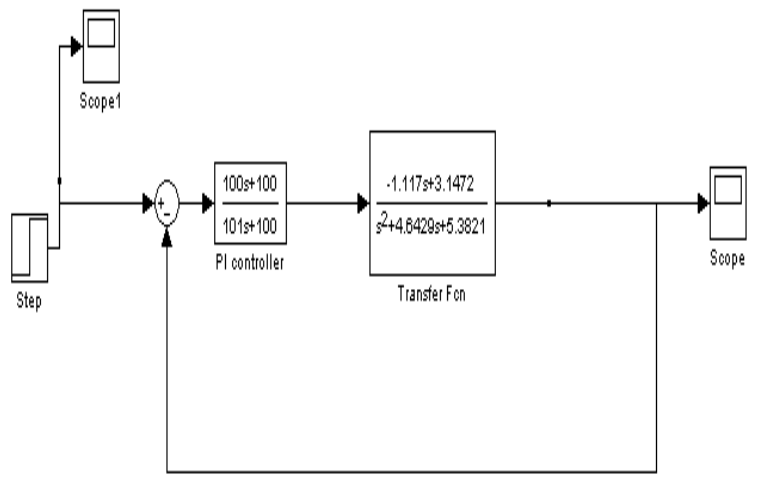

Fig. 5: Process model with PI controller

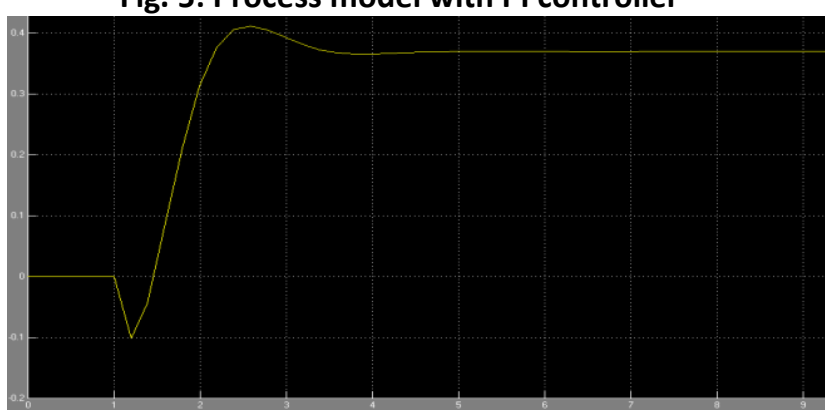

Fig. 6: Time response with PI controller

As can be seen from fig. 6, there is almost the same response as that of uncontrolled and $\mathrm{P}$ controller model.

The process with PID controller is shown in the fig. 7 and fig. 8 depicts the output of the process. The tuning of controller parameters is done by Zeigler \& Nichols method.

Here $K_{P}=0.1 \quad K_{I=} 0.2 \quad K_{D}=0.2$ 


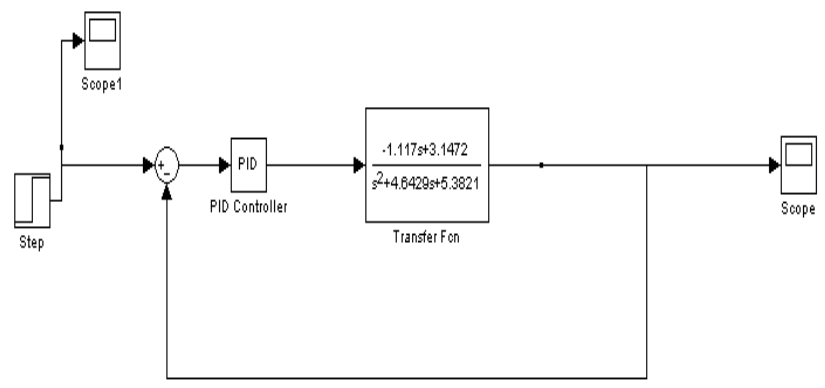

Fig. 7: Process model with PID controller

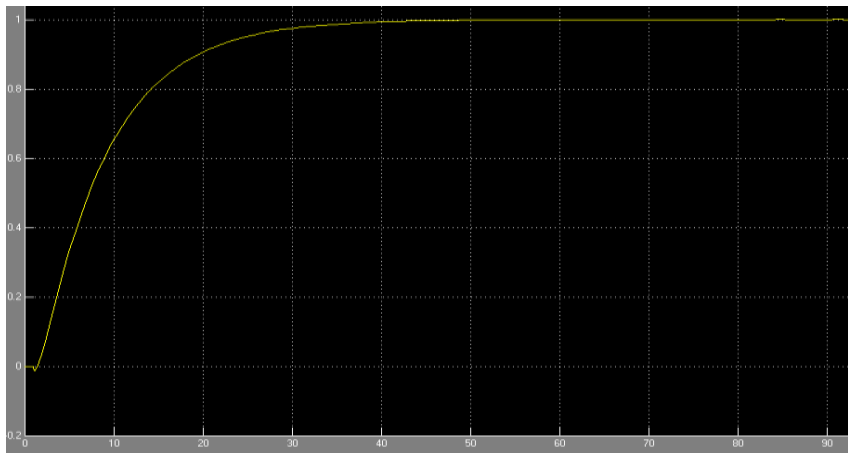

Fig. 8: Time response with PID controller

As can be observed from the fig. 8 ,

Rise time $\left(\mathrm{t}_{\mathrm{r}}\right)=20 \mathrm{sec} \quad$ Settling time $\left(\mathrm{t}_{\mathrm{s}}\right)=40 \mathrm{sec}$

Overshoot $=0 \% \quad$ undershoot $=0 \%$

There is almost negligible time delay and inverted response. So Fuzzy controller is used to reduce the rise time, settling time to almost negligible and also try to remove the time delay and inverted response.

The process is controlled by the fuzzy controller, is shown in fig. 9, and fig. 10 depicts the output of the process with fuzzy controller. For that the rules in FIS has been made and call them in simulink by the fuzzy logic controller.

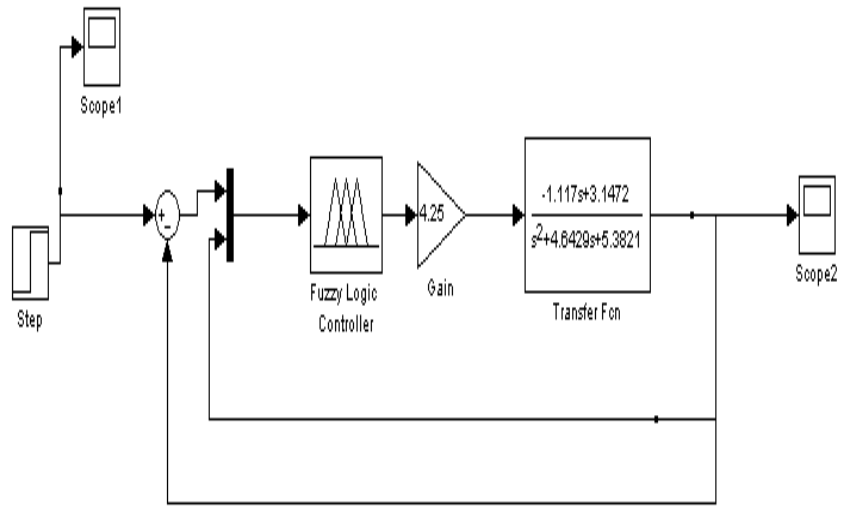

Fig. 9: Process model with fuzzy logic controller

The output response is shown below.

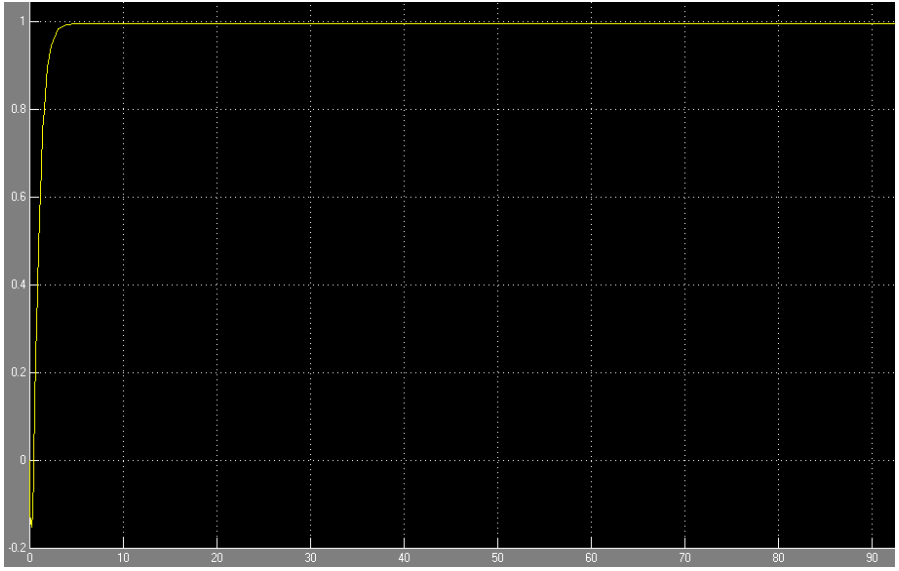

Fig. 10: Time response with fuzzy controller

As can be seen from fig. 10,

Rise time $\left(t_{r}\right)=3 \mathrm{sec}$ Overshoot $=0 \%$

Settling time $\left(t_{\mathrm{s}}\right)=4 \mathrm{sec}$ Undershoot $=0 \%$

There is no any time delay and also no any inverted response. All the limitations are reduced as compared to the PID controller.

In fig. 11, membership values of input 1 called "error" having three ranges low, medium and high is shown.

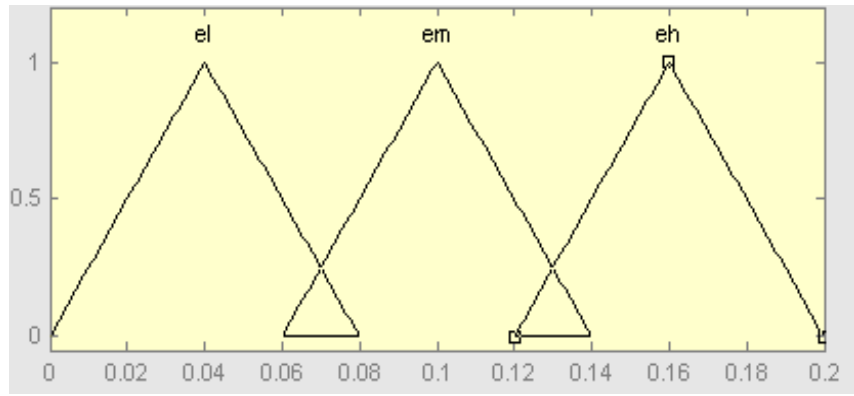

Fig. 11: Fuzzy membership sets of input '1' (error)

In fig. 12, the membership values of input 2 called "feedback" having the three ranges low, medium and high.

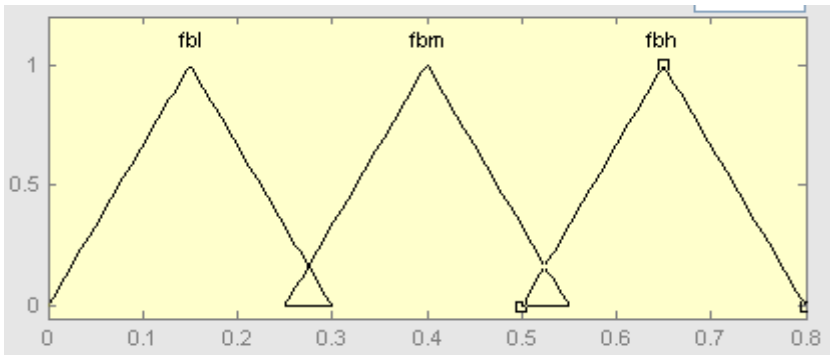

Fig. 12: Fuzzy membership sets of input '2' (feedback)

In fig. 13, membership values of "output" having the same ranges low, medium and high. 


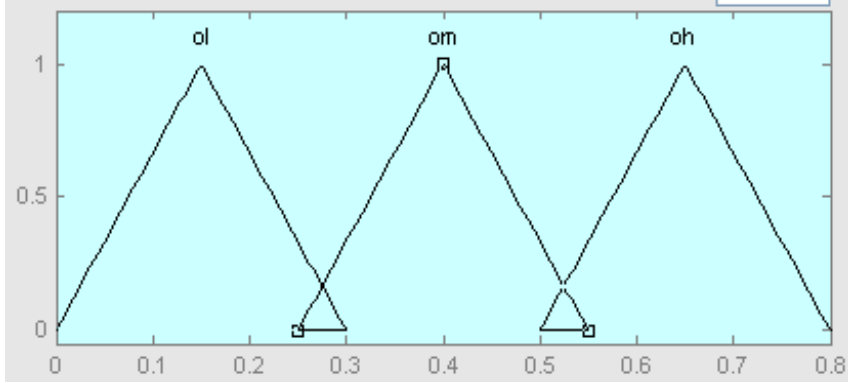

Fig. 13: Fuzzy membership sets of output (output)

In fig. 14, fuzzy if-then rules using mamdani fuzzy model are shown.

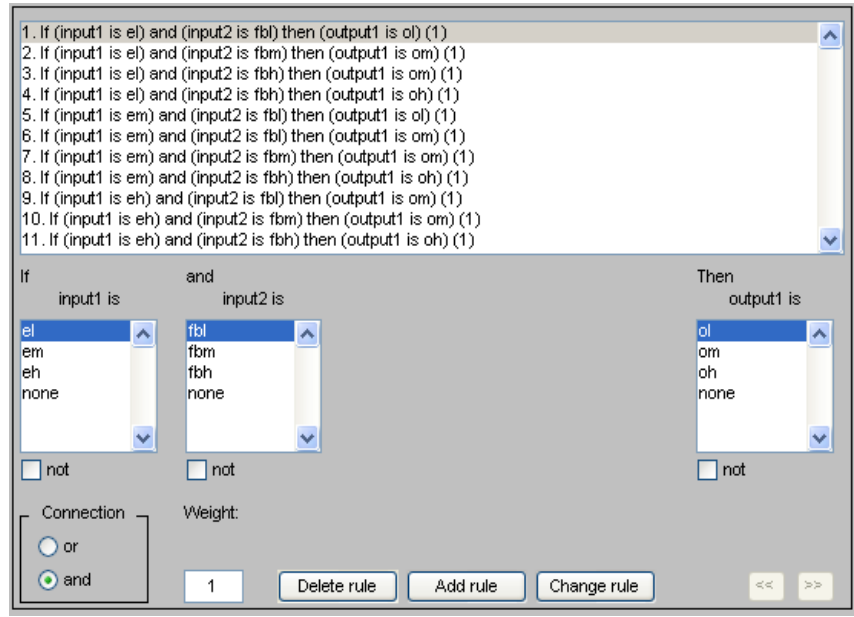

Fig. 14: Fuzzy If - then rules

Fig. 15 depicts the mesh analysis of the two inputs (error and feedback) and output.

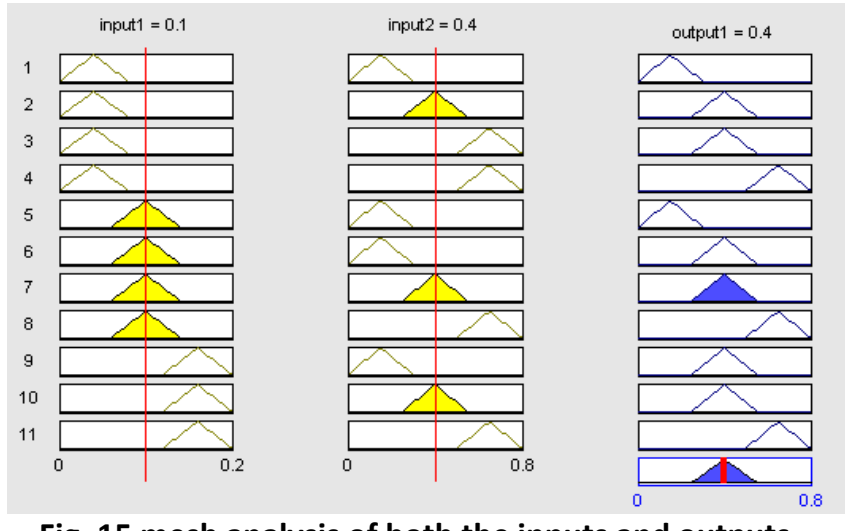

Fig. 15 mesh analysis of both the inputs and outputs

Fig. 16 depicts the surface view of the two inputs (error and feedback) and output.

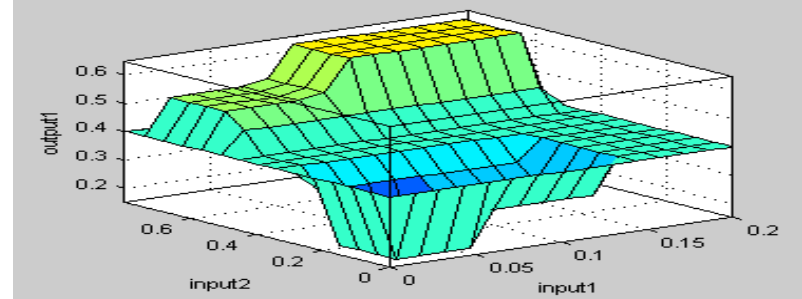

Fig.16 surface analysis of both inputs and outputs

\section{CONCLUSION}

When there is no control to the process, it generates an inverse response together with an overshoot and considerable delay time. But when the PID control is implemented to the process, the problems of inverse response, overshoot and delay time are controlled in the ongoing process and are removed considerably but then it was showing instability in terms of rise time and settling time. To overcome this instability in rise time \& in settling time a fuzzy logic controller has been used. The fuzzy control scheme helps to remove those delay times and the inverted response shown in graphs. Rise time and settling time are also reduced.

\section{REFERENCES}

[1] Schmidt, Lanny D. (1998). The Engineering of Chemical Reactions. New York: Oxford University Press. ISBN 019-510588-5

[2] Yung C. Shin, Chengying Xu "Intelligent Systems: Modeling, Optimization, and Control" CRC Press 2009.

[3] Fried land B., "Advanced Control System Design", Prentice Hall, New Jersey, 1996.

[4] Jaya, N. , Sivakumar, D. and Anandanatarajan, R. (2007) - Implementation of fuzzy gain scheduling of PID controllers for a two capacity interacting nonlinear process, paper presented at TIMA, January

[5] K.J. Astrom, \& T. Hagglund, "The future of PID control Control Engineering Practice”, pp.1163 -1175. 2001.

[6] Rajani K. Mudi, Chanchal Dey, \& Tsu - Tian Lee, "An improved auto - tuning scheme for PI controllers", Journal of science Direct ISA Transactions, 47: 45 - 52, 2008.

[7] Luis E. Zarate, Peterson Resende, \& M. Benjamin," A Fuzzy Logic and Variable Structure Base Controller for CSTR Control". Annual conference of IEEE, 2001.

[8] Xiao-Feng Li, Jian Sun, Hui-Yan Wu, Wei-Dong Zong, "Application of the fuzzy PID to the Power Plant", IEEE 2007.

[9] Yun Li,Kiam Heong Ang and Gregory C.Y.Chong, "PID Control System Analysis and Design-Problems, Remedies, and Future Directions". IEEE control system magazine, February 2006 pp. 32-41,2006

[10] Dimiter Driankov, Hans Hellendoorn, Michael Reinfrank, Fuzzy control 